\title{
Ablation of ATG4B Suppressed Autophagy and Activated AMPK for Cell Cycle Arrest in Cancer Cells
}

\author{
Pei-Feng Liu ${ }^{a} \quad$ Chien-Jen Hsu ${ }^{a, b} \quad$ Wei-Lun Tsaic,d Jin-Shiung Cheng ${ }^{c}$ \\ Jih-Jung Chene I-Fei Huang ${ }^{\mathrm{d}, \mathrm{f}}$ Ho-Hsing Tseng ${ }^{\mathrm{a}}$ Hsueh-Wei Changg,h,i \\ Chih-Wen Shu
}

aDepartment of Medical Education and Research, Kaohsiung Veterans General Hospital, Kaohsiung,

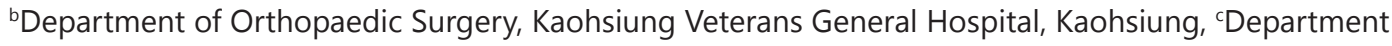
of Internal Medicine, Kaohsiung Veterans General Hospital, Kaohsiung, dSchool of Medicine, National Yang-Ming University, Taipei, eFaculty of Pharmacy, School of Pharmaceutical Sciences, National YangMing University, Taipei, 'Department of Pediatrics, Kaohsiung Veterans General Hospital, Kaohsiung, פDepartment of Biomedical Science and Environmental Biology, Kaohsiung Medical University, Kaohsiung, hDepartment of Medical Research, Kaohsiung Medical University Hospital, Kaohsiung, Institute of Medical Science and Technology, National Sun Yat-sen University, Kaohsiung, Taiwan

\section{Key Words}

ATG4B • AMPK • LKB1 • CCND1 • P27 kip1 $•$ Proliferation • Cell cycle • Autophagy

\begin{abstract}
Background/Aims: ATG4B is a cysteine protease required for autophagy, which is a cellular catabolic pathway involved in energy balance. ATG4B expression is elevated during tumor growth in certain types of cancer, suggesting that ATG4B is an attractive target for cancer therapy. However, little is known about the mechanisms through which ATG4B deprivation suppresses the growth of cancer cells. Methods: Cancer cells were transfected with either siRNA against ATG4B or an expression vector encoding wild-type ATG4BWT or encoding catalytic mutant $A T G 4 B^{C 74 A}$ to determine cell cycle progression by propidium iodide staining or by BrdU incorporation assay using flow cytometry. The GFP-MAP1LC3-II puncta and protein levels in the cells were determined by immunofluorescence and immunoblotting, respectively. Results: Knockdown of ATG4B blocked cell proliferation, particularly at the $G_{1}-S$ phase transition, in various cancer cells. Moreover, knockdown of ATG4B or overexpression of the ATG4B ${ }^{C 74 A}$ catalytic mutant reduced both autophagic flux and ATP levels and increased AMP-activated protein kinase (AMPK) phosphorylation in the cancer cells. Nevertheless, knockdown of ATG4B had only a minor effect on AMPK activation and $G_{1}$ phase arrest in liver kinase B1 (LKB1)-deficient or AMPK-inhibited cancer cells. Conclusion: These results imply that targeting ATG4B might inhibit autophagy and trigger the LKB1-AMPK energy-sensing pathway, resulting in tumor growth suppression.

P. Liu and C. Hsu contributed equally to this work.

Chih-Wen Shu

and Hsueh-Wei Chang

Kaohsiung Veterans General Hospital, Department of Medical Education and Research,

Taiwan, Department of Biomedical Science and Environmental Biology, Kaohsiung

Medical University, (Taiwan); E-Mail cwshu@vghks.gov.tw, changhw@kmu.edu.tw
\end{abstract}

KARGER 


\section{Introduction}

Macroautophagy (hereafter referred to as autophagy) is a selective clearance mechanism that degrades abnormal cellular components and generates ATP in eukaryotic cells experiencing starvation conditions. Autophagy-related (ATG) genes are required for the presence of autophagy machinery in cells. ATG4 is a cysteine protease that hydrolyses ATG8 at the C-terminal region to expose a glycine residue that can then conjugate with phosphatidylethanolamine (ATG8-II), an action that is required for autophagosome formation [1]. The mammalian orthologs of yeast ATG4 include ATG4A (autophagin-2), ATG4B (autophagin-1), ATG4C (autophagin-3) and ATG4D (autophagin-4) [2]. Among ATG4 members, ATG4B is the most potent and has a wide range of proteolytic activities on ATG8 orthologs in mammalian cells; these orthologs include MAP1LC3 (A, B and C) and GABARAP (GABARAP, GABARAPL1 and GABARAPL2) subfamilies [3-5]. ATG4B is also required for the deconjugation of MAP1LC3-II from non-autophagosomes or from autophagosomes to upregulate the autophagy machinery [6]. Crystal structure results show that the autoinhibitory N-terminal tail of ATG4B can be stabilized by non-substrate MAP1LC3, facilitating its targeting of membrane-bound MAP1LC3-II [7]. A recent study indicated that the phosphorylation of ATG4B at Ser383 and Ser392 is induced to increase proteolytic activity, thus delipidating membrane-bound MAP1LC3-II in starved cells [8]. These results suggest that tight regulation of ATG4B is crucial for the deconjugation of membrane-bound MAP1LC3-II and the facilitation of autophagy.

The association of ATG4B with cancer has been recently reported [9-11]. In colorectal cancer patients, ATG4B is highly expressed in tumor cells, whereas it is minimally expressed in adjacent normal cells [10]. Likewise, ATG4B and its family members are upregulated in $\mathrm{CD}^{+}{ }^{+}$chronic myeloid leukemia (CML) patients, resulting in drug resistance [11]. Silencing ATG4B significantly suppresses colorectal cancer cell growth and sensitizes HER2-positive breast cancer cells to treatment with trastuzumab [12]. Overexpression of the dominant negative mutant $\mathrm{ATG}_{4} \mathrm{~B}^{\mathrm{C} 74 \mathrm{~A}}$ decreases cell viability in hepatocellular carcinoma [13] and sensitizes prostate cancer C42 and PC3 cells to chemotherapeutic drugs and radiation [14]. However, Atg4b-knockout mice have impaired autophagic flux that reduces protein secretion and causes inner ear imbalance and bone resorption $[15,16]$. The mice lacking ATG4B are perfectly viable and do not have serious abnormalities [17], implying that ATG4B might be an attractive target for cancer therapy.

Autophagy inhibition has been found to reduce ATP levels in cells [18, 19]. AMPactivated protein kinase (AMPK) is activated in cells with low ATP levels to arrest cell cycle progression and promote ATP production [20], suggesting that autophagy inhibition may stimulate a positive feedback loop for AMPK activation to suppress cell proliferation. Liver kinase B1 (LKB1) is the main upstream kinase required for phosphorylation at Thr172 in the activation loop of AMPK [21]. Thus, LKB1-AMPK serves as a nutrient and energy sensor to balance ATP levels in cells. Moreover, the mechanistic target of rapamycin complex 1 (mTORC1) is a downstream effector of AMPK and comprises mTOR and several binding partners, such as the regulatory associated protein of mTOR (raptor), mammalian lethal with SEC13 protein 8 (MLST8) and proline-rich AKT1 substrate 1 (PRAS) [22]. 4EBP1 and ribosomal S6 kinase are recruited by raptor to mTORC1 for their activation and modulate cell proliferation [23]. AMPK phosphorylates raptor and triggers a 14-3-3 association with the phosphorylated raptor to block mTORC1's ability to bind to its substrates, which in turn inhibits cell proliferation [24]. In addition, AMPK can directly phosphorylate and stabilize $\mathrm{p} 27^{\mathrm{kip} 1}$ or indirectly increase the expression of $\mathrm{p} 27^{\mathrm{kip} 1}$ [25], which is a negative regulator of cell cycle progression. These results indicate that AMPK serves as a metabolic checkpoint for cell growth based on cell energy status.

To date, little is known about the mechanisms of targeting ATG4B for tumor suppression. Thus, we aimed to determine the mechanisms by which ATG4B affects cancer cell proliferation. In this study, ATG4B knockdown blocked the $\mathrm{G}_{1} / \mathrm{S}$ phase transition in several types of cancer cells, including neuroblastoma H4 cells, breast cancer MCF-7 cells and prostate cancer 


\section{Cellular Physiology Cell Physiol Biochem 2017;44:728-740 \begin{tabular}{l|l|l} 
and Biochemistry 10.1159/000485286 & $\begin{array}{l}\text { C) } 2017 \text { The Author(s). Published by S. Karger AG, Basel } \\
\text { www.karger.com/cpb }\end{array}$ \\
\hline
\end{tabular}}

Liu et al.: AMPK Activation in ATG4B-Knockdown Cancer Cells

PPC1 cells. Furthermore, silencing ATG4B or overexpressing the catalytic mutant ATG4B ${ }^{\mathrm{C} 74 \mathrm{~A}}$ reduced ATP levels and increased the phosphorylation of AMPK and raptor in the cancer cells, whereas ATG4B knockdown had little or no effect on AMPK phosphorylation and cell cycle arrest in LKB1-deficient cells. Our results suggest that the knockdown of ATG4B may activate the LKB1-AMPK energy-sensing pathway and thereby suppress cancer cell proliferation.

\section{Materials and Methods}

\section{Plasmids and siRNA transfection}

Human neuroblastoma H4 cells, breast cancer MCF-7 and MDA-MB-231 cells, prostate cancer PPC1 cells, and cervical cancer HeLa cells were cultured in Dulbecco's modified Eagle's medium (DMEM) (Invitrogen, 12100-046) containing 10\% fetal bovine serum, penicillin (100 U/ml), and streptomycin (100 $\mathrm{mg} / \mathrm{ml}$ ). The cells were reverse transfected with scrambled siRNA (Life Technologies, 12935-112) or siRNA against ATG4B (Life Technologies, 20218, s23245, s23246) for $72 \mathrm{~h}$ using the transfection reagent RNAiMAX (Life Technologies, 13778-150). For plasmid transfection, the cells were transfected with $2 \mu \mathrm{g}$ of cherrytagged $\mathrm{ATG}^{\mathrm{W}} \mathrm{B}^{\mathrm{WT}}$ or $\mathrm{ATG}^{\mathrm{C}} \mathrm{B}^{\mathrm{C} 74 \mathrm{~A}}$ using Lipofectamine 2000 transfection reagent (Invitrogen, 11668-027) for $24 \mathrm{~h}$. The cells were harvested for either protein expression analysis by immunoblotting or cell cycle analysis by flow cytometry.

\section{Generation of stable cell line}

shRNAs against the 3'UTR of ATG4B (TRCN0000073801) were obtained from The RNAi Consortium (TRC, Taiwan). The plasmid ( $2 \mu \mathrm{g}$ ) was mixed with Lipofectamine 2000 (Life Technologies, 11668-027) and transfected into 293FT cells $\left(1 \times 10^{6}\right.$ cells $)$. The supernatant was harvested after 2 days and centrifuged to remove cell debris and to collect virus particles used to infect $\mathrm{H} 4$ cells. The infected $\mathrm{H} 4$ cells were selected with puromycin $(1 \mu \mathrm{g} / \mathrm{ml})$ for 14 days to obtain ATG4B-knockdown cells. The cells lacking ATG4B expression were further transfected with an expression vector encoding cherry or cherry-tagged ATG4B ${ }^{\mathrm{WT}}$ and ATG4B ${ }^{\mathrm{C} 74 \mathrm{~A}}$. The cells were selected with geneticin $(800 \mu \mathrm{g} / \mathrm{ml}$, Thermo Fisher Scientific, 10131035), and the cherry-expressing cells were purified with a BD FACSAria ${ }^{\mathrm{TM}}$ III sorter (Becton Dickinson).

\section{Immunoblotting}

The silenced cells were treated with or without the autophagy inhibitors BafA1 (Millipore, 196000) or CQ (Sigma-Aldrich, C6628) and then lysed in RIPA buffer (1\% NP40, $50 \mathrm{mM}$ Tris- $\mathrm{HCl}$ [pH 7.5], $150 \mathrm{mM} \mathrm{NaCl}$, $0.25 \%$ sodium deoxycholate, $1 \%$ sodium dodecyl sulfate (SDS), protease inhibitor cocktail and phosphatase inhibitor). The proteins were separated by SDS-PAGE and transferred onto nitrocellulose membranes for immunoblotting. The membrane were rinsed with Odyssey Blocking Buffer (Li-COR, 927-50000) and then incubated overnight at $4^{\circ} \mathrm{C}$ with primary antibodies against ATG4B (A2981), MAP1LC3 (L7543) and ACTB (A5441) (purchased from Sigma-Aldrich); p2 $7^{\mathrm{kip} 1}$ (purchased from Santa Cruz Biotechnology); and CCND1 (cyclin D1, 2926), pThr172-AMPK (5536), AMPK (2532), pSer792-raptor (2083) and raptor (2280) (purchased from Cell Signaling Technology). The proteins were probed with an HRP-labeled secondary antibody (Santa Cruz Biotechnology, sc-2004 or sc-2005) and detected with an ECL reagent. The membrane was scanned and analyzed for protein expression levels using the ChemiDoc XRS Imaging System (Bio-Rad). The protein levels were measured using ImageJ software.

\section{Fluorescence microscopy}

The cells harboring the GFP-MAP1LC3 expression vector were seeded on gelatin-coated glass slides for $24 \mathrm{~h}$. The cells were transfected with siRNA for $72 \mathrm{~h}$, fixed with $3.7 \%$ paraformaldehyde at room temperature for $15 \mathrm{~min}$ and then washed with PBS three times prior to observation. The GFP-MAP1LC3 puncta were observed under a fluorescence microscope, and the number of GFP-MAP1LC3 puncta in each cell was counted for quantification.

Cell viability assay and ATP measurement

To assay cell viability, the cells were seeded in a 96-well white plate (Greiner, 655083) and transfected with $5 \mathrm{nM}$ scrambled siRNA or pooled siRNA against ATG4B for various time points. CellTiter-Glo (Promega, 
Liu et al.: AMPK Activation in ATG4B-Knockdown Cancer Cells

G7572) containing luciferase and D-luciferin was then added to the cells, and cellular ATP concentrations were measured to determine cell viability. In addition, cells were rinsed with PBS and harvested to count the number of cells. Equal amounts of cells were mixed with CellTiter-Glo in a 96-well white plate to measure ATP levels. The luminescent signal was detected using a Fluoroskan Ascent FL reader (Thermo Fisher Scientific). The results are expressed as cell viability and ATP level relative to the control cells.

\section{Clonogenic assay}

The cells were seeded in 6-well plates and transfected with scrambled siRNA or pooled siRNA against ATG4B. The culture medium was changed every 3 days for 2-3 weeks. The cell colonies were fixed and stained with $0.25 \%$ crystal violet at room temperature for $30 \mathrm{~min}$ as previously reported [10]. The stained cells were washed with PBS to remove the background, enabling observation of the colonies. The colonies over $1 \mathrm{~mm}$ were counted and quantified with Prism 5.0 (Graph-Pad).

\section{Flow cytometry for cell cycle and proliferation analyses}

To analyze the cell cycle distribution, the siRNA-transfected or compound C (Millipore, 171260)-treated cells were rinsed with PBS and then fixed in $75 \%$ ethanol at $-20^{\circ} \mathrm{C}$ overnight. The fixed cells were then stained with propidium iodide ( $50 \mu \mathrm{g} / \mathrm{ml}$, Sigma-Aldrich) and RNase A ( $25 \mu \mathrm{g} / \mathrm{ml}$, Sigma-Aldrich) on ice for 30 min. The cell cycle proportions of stained cells were analyzed using a FACScan flow cytometer (Becton Dickinson). To precisely quantitate the S phase population of the cells, the cells were treated with $10 \mu \mathrm{M}$ BrdU for $1.5 \mathrm{~h}$ and then fixed for further probing with a fluorescein isothiocyanate (FITC)-conjugated antiBrdU antibody and 7-aminoactinomycin D (7AAD). The S phase proportion of stained cells was determined using a FACScan flow cytometer (Becton Dickinson) and analyzed by FlowJo software (Tree Star).

\section{Statistical analysis}

All the data are expressed as the mean \pm SEM from three individual experiments. The statistical analysis was performed using a nonparametric 2-tailed Student's t-test with Prism 5.0 (Graph-Pad). p values lower than 0.05 were considered significant.

\section{Results}

Knockdown of ATG4B induced cell cycle arrest in various cancer cells

Based on reports of the oncogenic roles of ATG4B in patients with colorectal cancer and CML $[10,11]$, we confirmed a role for ATG4B in tumor growth in various human cancer cells, including human neuroblastoma H4 cells, breast cancer MCF-7 cells and prostate cancer PPC1 cells. Silencing ATG4B with three individual or pooled siRNAs against ATG4B suppressed ATG4B expression (Fig. 1A). Consistent with the knockdown efficiency shown in the immunoblotting results, silencing ATG4B resulted in lower cell viability in the cancer cells (Fig. 1B). To minimize the off-target effects of siRNAs targeting ATG4B, pooled siRNAs were used to knockdown ATG4B for subsequent experiments. The colony formation of cells transfected with pooled siRNA against ATG4B was considerably less in these cancer cells (Fig. 1C and 1D).

Cell cycle analysis was used to evaluate whether the tumor-suppressive effects of silencing ATG4B occurred because of cell cycle arrest or cell death. Knockdown of ATG4B in cancer cells increased the percentage of cells in $G_{1}$ phase and accordingly decreased the percentage of cells in S phase, whereas ATG4B knockdown had little to no effect on the

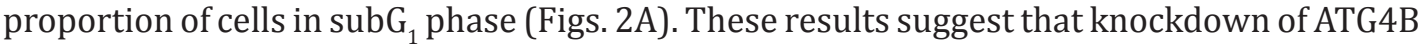
led to cell cycle arrest in cancer cells rather than inducing cell death. To more precisely measure the population of cells in S phase, a BrdU incorporation assay was employed for the cancer cells silenced by siRNA against ATG4B (Fig. 2B). The ATG4B-knockdown cancer cells had significantly fewer BrdU-positive cells (Fig. 2C). Likewise, cells silenced with siRNA against ATG4B were further stained with BrdU-7AAD to measure the percentage of cells in $G_{1}$ and $S$ phases (Fig. 2D). The ratio of $S / G_{1}$ phase cells was greatly reduced in ATG4Bsilenced cells, supporting the idea that ATG4B might be required for cell cycle regulation, particularly in the transition from $G_{1}$ to $S$ phase. 
Fig. 1. Effects of silencing ATG4B on tumor growth in various cancer cells. (A) Scrambled siRNA ( $5 \mathrm{nM}$, siCtrl) or three individual siRNAs against ATG4B ( 5 nM, siATG4B) were transfected into human neuroglioma $\mathrm{H} 4$ cells, breast cancer MCF-7 cells, and prostate cancer PPC1 cells for $72 \mathrm{~h}$. The cells were harvested for immunoblotting to verify the knockdown efficiency of ATG4B. (B) The cell viability of ATG4B-silenced (siATG4B) cancer cells was measured with CellTiterGlo every 24 h until 96 h. Scrambled siRNA (siCtrl) was used as a control. (C) The colony formation of ATG4Bsilenced cancer cells was counted to determine the tumor-suppressive effect of ATG4B knockdown. Representative results are shown. (D) The quantitative results are expressed as the mean \pm SEM from three individual experiments. ${ }^{* * *} \mathrm{p}<0.001$ vs. scramble siRNA (siCtrl).

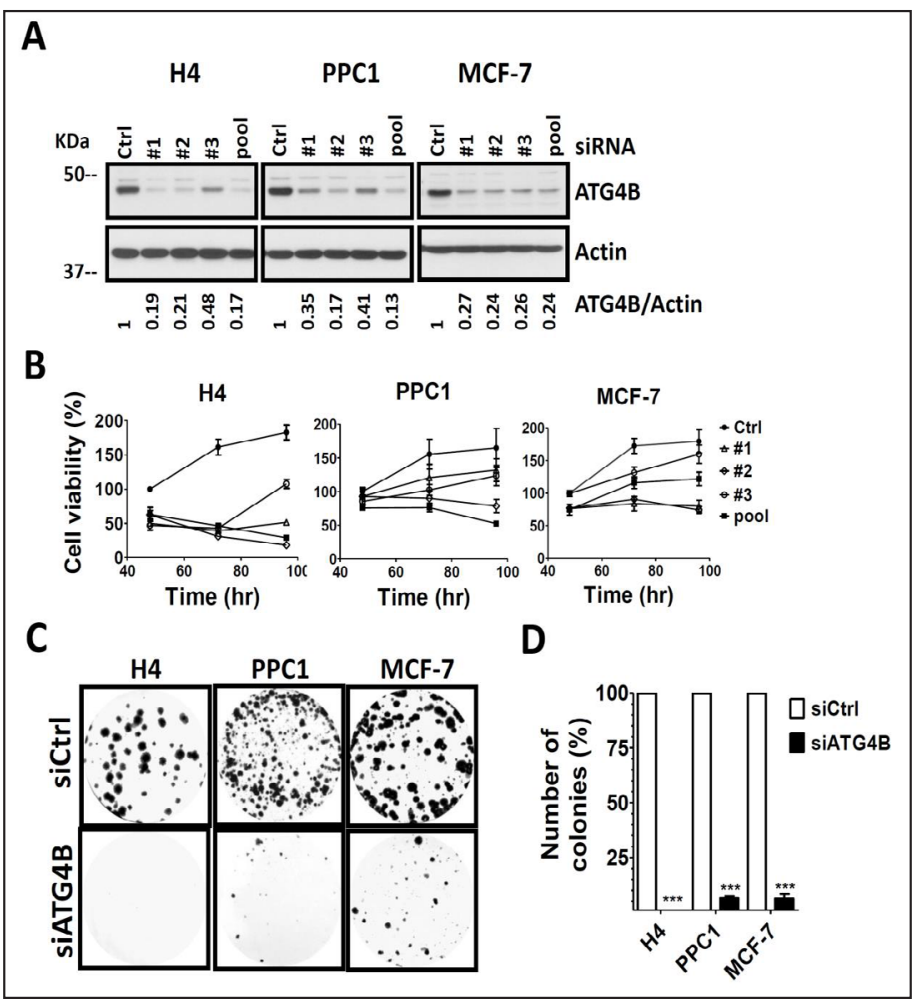

Silencing ATG4B diminished autophagy and activated AMPK in cancer cells

Autophagy inhibition also resulted in a reduction in ATP levels, which may activate the AMPK energy-sensing pathway for regulating the cell cycle and balancing ATP levels [18, 24]. ATG4B is a key modulator required by autophagy machinery. We observed that GFPMAP1LC3-II puncta were increased in ATG4B-silenced cancer cells (Fig. 3A), which was likely due to reduction in the delipidation of MAP1LC3-II in membranes [6]. With the combination of bafilomycin A1 (BafA1) and the siRNA against ATG4B, the changes in MAP1LC3-II were fewer than those in the control cells, indicating that autophagic flux was diminished in ATG4B-silenced cells (Fig. 3B and 3C). To determine the effects of ATG4B knockdown on ATP levels, we examined ATP levels in cells silenced with siRNA against ATG4B. Knockdown of ATG4B reduced the ATP levels in the cells (Fig. 3D). Moreover, the phosphorylation of AMPK at Thr172 and of raptor at Ser792 was increased in ATG4B-silenced cells (Figs. 3E). The levels of $\mathrm{p} 27^{\mathrm{kip} 1}$ and cyclin D1 (CCND1), downstream effectors of AMPK and mTORC1 that are involved in cell cycle regulation at the $\mathrm{G}_{1}-\mathrm{S}$ phase transition, were also accordingly increased and decreased, respectively.

Because AMPK can increase p27 $7^{\mathrm{kip} 1}$ expression and stability [25], p27 ${ }^{\mathrm{kip} 1}$ expression was further evaluated in ATG4B-silenced cells with proteasomal or lysosomal inhibitors (Fig. 3F). The increased p27 ${ }^{\text {kip } 1}$ in ATG4B-knockdown cells was modestly augmented by proteasomal or lysosomal inhibitors (Fig. 3G), suggesting that silencing ATG4B might increase both the expression and stability of p $27^{\mathrm{kip}} 1$. More importantly, compound $\mathrm{C}$, an AMPK inhibitor, blunted the increased number of cells in $\mathrm{G}_{1}$ phase in ATG4B-silenced cancer cells (Fig. 3H), indicating that AMPK plays a role in cell cycle modulation in cells when silencing ATG4B.

To further determine whether the LKB1-AMPK energy-sensing pathway is involved in $\mathrm{G}_{1}$ phase arrest in ATG4B-silenced cells, the human cervical cancer HeLa cell line, an LKB1 null cell line, was transfected with scrambled siRNA or siRNA against ATG4B and ATP levels, AMPK phosphorylation and cell cycle distribution were measured (Fig. 4A-C). Although the ATP level was reduced in ATG4B-silenced HeLa cells, the phosphorylation of AMPK and 
Fig. 2. Silencing ATG4B inhibited the G1/S phase transition in human cancer cells. (A) Human neuroblastoma H4 cells, breast cancer MCF-7 cells, and prostate cancer cells were transfected for $72 \mathrm{~h}$ with $5 \mathrm{nM}$ pooled scrambled siRNA (siCtrl) or siRNA against ATG4B (siATG4B). The cells were harvested to analyze the cell cycle using flow cytometry. The cell cycle distribution was analyzed and quantified with FlowJo software. (B) The ATG4B-silenced

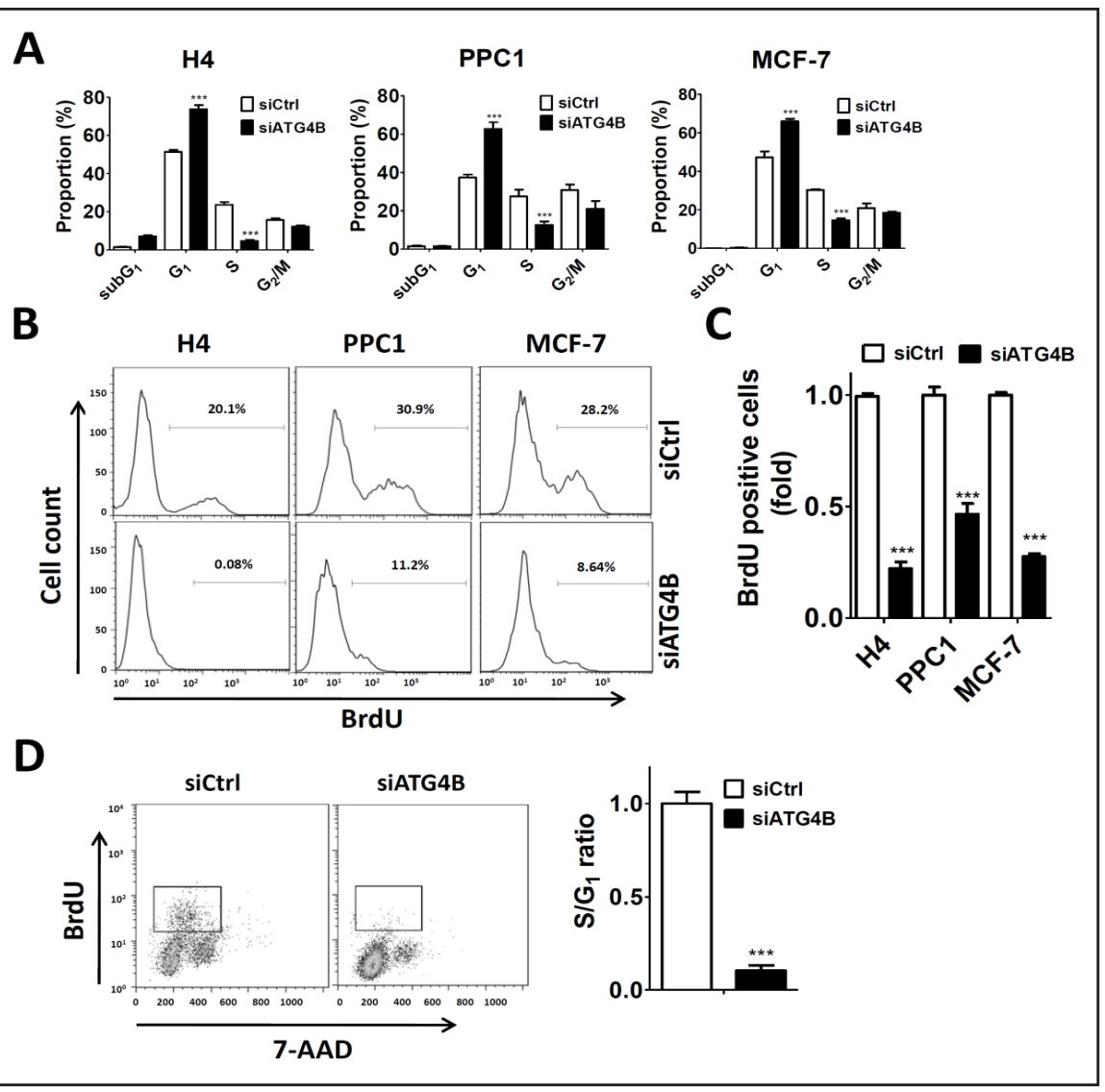
cancer cells as in

(A) were incubated with $10 \mu \mathrm{M}$ BrdU for $2 \mathrm{~h}$ prior to harvest and analysis of BrdU-positive cells by flow cytometry, which indicates the cell population in S phase of the cell cycle. (C) The percentage of BrdU-positive cells was quantified. (D) Human neuroglioma H4 cells silenced with siRNA against ATG4B were stained with BrdU/7-AAD for cell cycle analysis using flow cytometry. The BrdU-positive cells were labeled (tangle, left panel), and the ratio of cells in S/G1 phase is quantified in the right panel. The results are expressed as the mean \pm SEM from three individual experiments. ${ }^{* * *} \mathrm{p}<0.001$ vs. scramble siRNA (siCtrl).

raptor was not elevated. Consistently, knockdown of ATG4B did not alter colony formation or cell cycle distribution compared to the cells with scrambled siRNA (Fig. 4C). In addition, HeLa cells are phenotypically null cells for p53, which negatively regulates cell cycle progression mainly through its transcriptional target, p21 [26]. We further evaluated whether p53 is involved in cell cycle arrest in ATG4B-silenced cells. Knockdown of ATG4B in H4 and MCF-7 cells did not alter the phosphorylation of p53 or the expression of its downstream target, p21 (data not shown). Moreover, silencing ATG4B inhibited cell growth and arrested the cell cycle at $\mathrm{G}_{1}$ phase in MDA-MB-231 cells, which bear the p53 mutant R280K [27] (Fig. 4D and $4 \mathrm{E}$ ). In addition, silencing $\mathrm{LKB} 1$ diminished $\mathrm{G}_{1}$ phase arrest caused by the knockdown of ATG4B in cells (Fig. 4F), suggesting that the LKB1-AMPK energy-sensing pathway might be involved in cell cycle arrest at $G_{1}$ phase in certain cancer cells when ATG4B is silenced by siRNA.

\section{ATG4B ${ }^{C 74 A}$ catalytic mutant increased the phosphorylation of AMPK and arrested cell cycle progression}

To further determine if the proteolytic activity of ATG4B is required for the proliferation of cancer cells, H4 cells were stably infected with shRNA against the 3'UTR of ATG4B to knockdown endogenous ATG4B and were reconstituted with cherry-tagged expression vectors encoding wild-type (ATG4B ${ }^{\mathrm{WT}}$ ) or catalytic mutant (ATG4B ${ }^{\mathrm{C} 74 \mathrm{~A}}$ ) proteins (Fig. 5). In 
Fig. 3. Effects of the AMPK energy-sensing pathway in ATG4Bsilenced cells. (A) The cells stably harboring GFP-MAP1LC3 were transfected with siRNA against ATG4B for 72 $h$ to observe GFP-MAP1LC3 puncta under a fluorescence microscope. The number of GFP-MAP1LC3 puncta in each cell are quantified in the right panel. Bar: $10 \mu \mathrm{m}$. (B) The cells were harvested to determine the protein levels of ATG4B and MAP1LC3 by immunoblotting; ACTB was used as the control. (C) The net MAP1LC3-II in cells with and without BafA1 was used to measure the autophagic flux. (D) Human neuroglioma $\mathrm{H} 4$ cells were transfected with $5 \mathrm{nM}$ pooled scrambled siRNA or siRNA against ATG4B for 48 h. Equal amounts of cells were used to determine the ATP level. (E) The knockdown cells were harvested

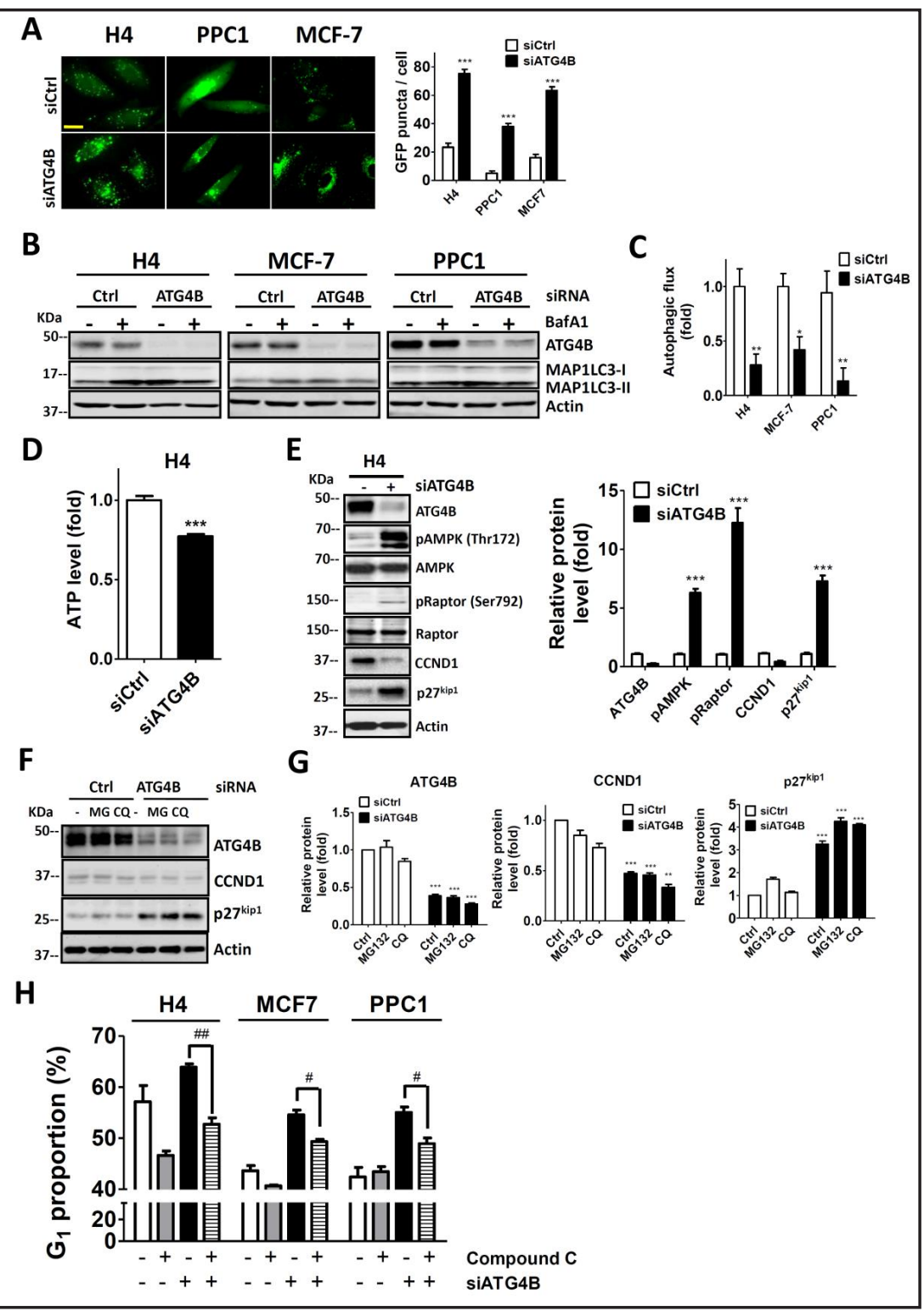
to measure the phosphorylation of AMPK and raptor and the expression of CCND1 and p27 by immunoblotting (left panel). Protein phosphorylation and expression are quantified in the right panel. (F) $\mathrm{H} 4$ cells were treated with 5 $\mathrm{nM}$ siRNA against ATG4B for $48 \mathrm{~h}$, followed by treatment with the proteasome inhibitor MG132 (2 M) or the lysosome inhibitor CQ $(20 \mu \mathrm{M})$ for $16 \mathrm{~h}$. The cells were harvested to determine the downstream effectors of AMPK for the $\mathrm{G}_{1} / \mathrm{S}$ phase transition by immunoblotting, including cyclin D1 and p27 $7^{\mathrm{kip} 1}$. ACTB was used as the control. (G) Quantitative results are expressed as the mean \pm SEM. (H) The cells were transfected with siRNA against ATG4B in the presence or absence of compound $\mathrm{C}(1 \mu \mathrm{M})$ and harvested for cell cycle analysis by flow cytometry. ${ }^{*} \mathrm{p}<0.05,{ }^{* *} \mathrm{p}<0.01,{ }^{* * *} \mathrm{p}<0.001$ vs. scramble siRNA (siCtrl). ${ }^{*} \mathrm{p}<0.05,{ }^{\# \#} \mathrm{p}<0.01$ vs. siATG4B alone.

contrast to cells with ATG4B ${ }^{\mathrm{WT}}$ expression vector, colony formation was greatly decreased in cells with shRNA against the 3'UTR of ATG4B or reconstituted with ATG4B ${ }^{\mathrm{C} 74 \mathrm{~A}}$ expression vector (Fig. 5A). Likewise, the proportions of $G_{1}$ and BrdU-positive cells in ATG4B ${ }^{\text {C74A }}$ reconstituted cells were elevated and diminished, respectively (Figs. 5B, 5C). Furthermore, we evaluated whether the molecular signaling mentioned above is involved in cell cycle 


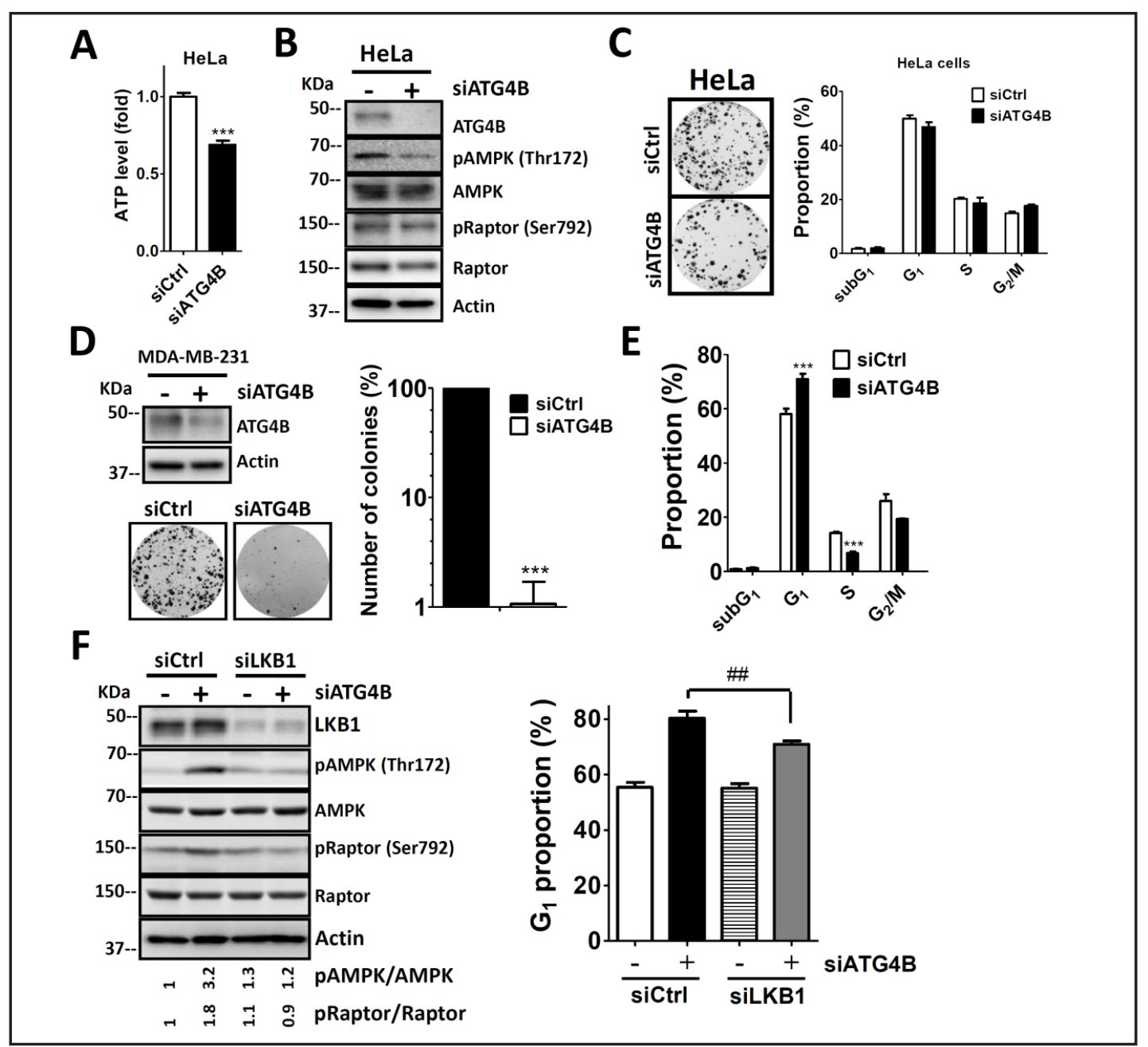

Fig. 4. Involvement of LKB1 in silencing ATG4B-mediated cell cycle arrest. (A) Scrambled (-) or ATG4B (+) siRNA ( $5 \mathrm{nM}$ ) was transfected into HeLa cells and LKB1-deficient cells for $48 \mathrm{~h}$. Equal amounts of cells were collected to measure the ATP level. (B) ATG4B-silenced HeLa cells were collected to examine the protein levels of pAMPK (Thr172) and p-raptor (Ser792) by immunoblotting, and the knockdown efficiency of ATG4B was examined. (C) HeLa cells were transfected with $5 \mathrm{nM}$ scramble (-) or ATG4B (+) siRNA for 72 $\mathrm{h}$ to determine the effects on tumor growth by clonogenic assay and cell cycle distribution. (D) Human breast cancer MDA-MB-231 cells and P53 mutant cells were treated with siRNA against ATG4B for $72 \mathrm{~h}$. The growth of the knockdown cells was determined by clonogenic assay and (E) flow cytometry. (F) H4 cells were transfected with 5 nM siRNA against LKB1 for $48 \mathrm{~h}$, followed by transfection with siRNA against ATG4B. The knockdown efficiency was verified by immunoblotting, and the proportion of the cells in $G_{1}$ phase was analyzed by flow cytometry. ${ }^{* * *}$ p $<0.001$ vs. scramble siRNA (siCtrl). ${ }^{\# \#}$ p $<0.01$ vs. siATG4B alone.

arrest in ATG4B ${ }^{\mathrm{C} 74 \mathrm{~A}}$-reconstituted cells. Similar to ATG4B-silenced cells, the level of GFPMAP1LC3-II puncta was increased in ATG4B ${ }^{\mathrm{C} 74 \mathrm{~A}}$-reconstituted cells (Fig. 5D), indicating the dominant role of ATG4B in the delipidation of MAP1LC3-II from non-autophagosomes or autophagosomes to facilitate autophagy as previously reported [6]. AMPK phosphorylation was significantly elevated in stable knockdown cells and ATG4B ${ }^{\text {C74A }}$-expressing cells compared with ATG4B ${ }^{\text {WT }}$-expressing cells (Fig. 5E). The downstream effectors of AMPK, including raptor phosphorylation and $\mathrm{p} 27^{\mathrm{kip} 1}$ expression, were increased accordingly in stable knockdown and $\mathrm{ATG}_{4} \mathrm{~B}^{\mathrm{C} 74 \mathrm{~A}}$-expressing cells. In contrast to ATG4B ${ }^{\mathrm{WT}}$-reconstituted cells, the ATP level was decreased in ATG4B ${ }^{\mathrm{C} 74 \mathrm{~A}}$-reconstituted cells (Fig. 5F). Treatment with the AMPK inhibitor compound $C$ partially blunted the increase in the number of cells in $G_{1}$ 


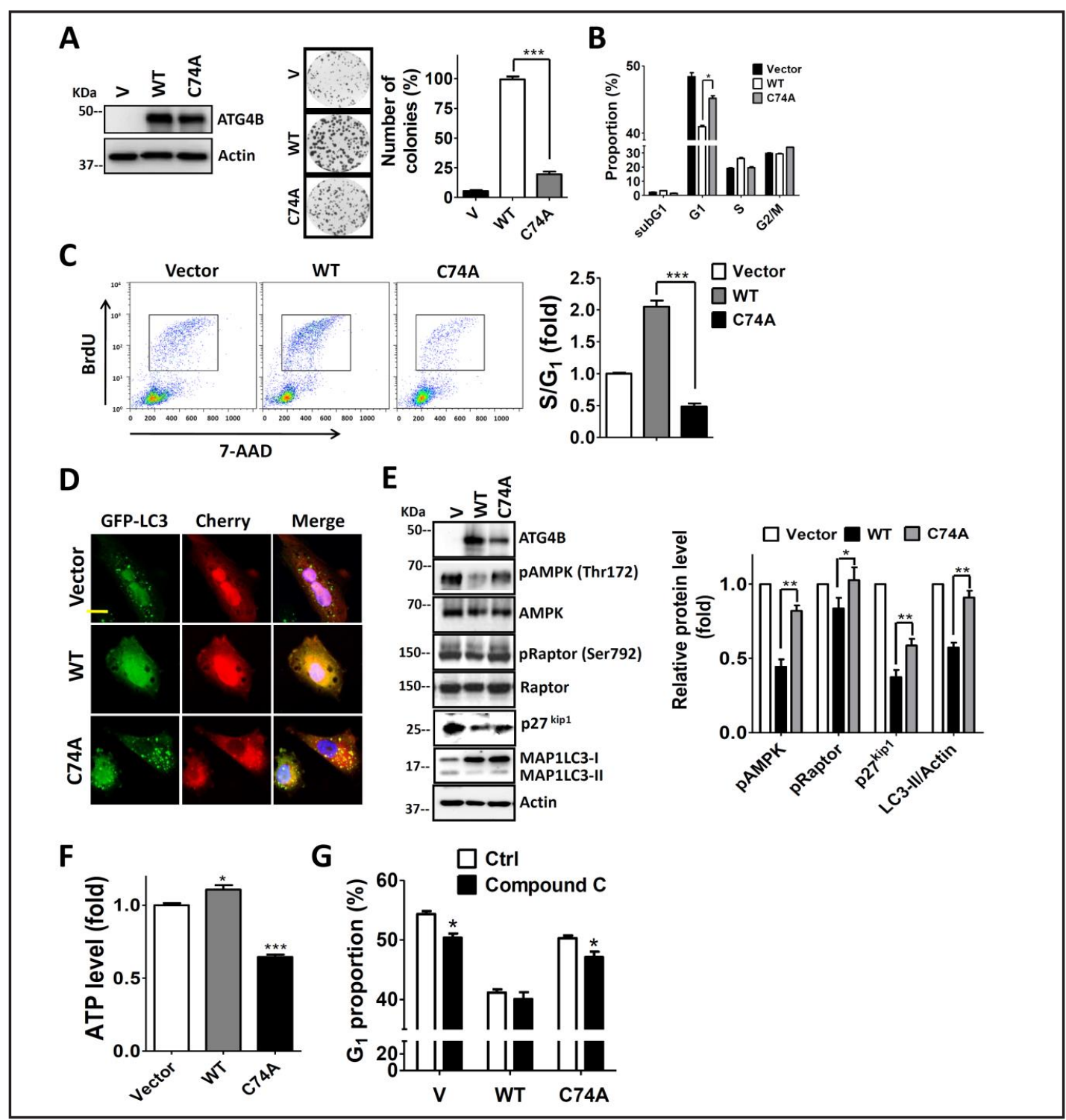

Fig. 5. Effects of the ATG4B catalytic mutant on AMPK activation and tumor growth. H4 cells stably harboring shRNA against the 3'UTR of ATG4B were reconstituted with cherry, ATG4B wild-type (WT)cherry or ATG4B C74A-cherry expression plasmids. (A) The cells were used for the clonogenic assay and (B) cell cycle distribution. (C) BrdU was incorporated into the cells, and the cells were then stained with 7AAD to observe the transition between $G_{1}$ and $S$ phases. (D) The cells in (A) were transfected with $1 \mu \mathrm{g}$ of GFP-MAP1LC3 for $36 \mathrm{~h}$ and fixed to observe the puncta under confocal microscopy. Bar: $10 \mu \mathrm{m}$. (E) The cells in (A) were harvested to determine the phosphorylation of AMPK and downstream effectors by immunoblotting, including phosphor-raptor and p27. The quantitative results are shown in the right panel. (F) Equal amounts of cells were collected to measure the ATP level. (G) The cells were treated with compound $C(1 \mu \mathrm{M})$ overnight to measure the proportion of cells in $G_{1}$ phase by flow cytometry. The results are expressed as the mean \pm SEM from three individual experiments. ${ }^{*} \mathrm{p}<0.05,{ }^{* *} \mathrm{p}<0.01,{ }^{* * *} \mathrm{p}<0.001$ vs. ATG4B wild-type (WT).

phase caused by stable knockdown of ATG4B or the ectopically expressed ATG4B ${ }^{\mathrm{C} 74 \mathrm{~A}}$ mutant (Fig. 5G). These results indicate that ATG4B ${ }^{\mathrm{C} 74 \mathrm{~A}}$ may inhibit autophagy, lower ATP levels and, in turn, activate AMPK for cell growth arrest, particularly in the $G_{1}-S$ phase transition in cancer cells. 


\section{Cellular Physiology Cell Physiol Biochem 2017;44:728-740

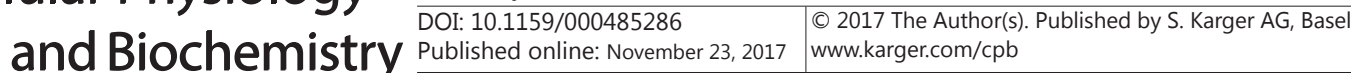 Liu et al.: AMPK Activation in ATG4B-Knockdown Cancer Cells}

\section{Discussion}

ATG4B is a cysteine protease required by autophagy machinery in mammalian cells. Elevated expression of ATG4B has been observed in colorectal cancer and CML patients [10, $11]$. Knockdown of ATG4B suppresses tumor growth in vitro and in vivo [10, 11]. However, little is known about the detailed mechanisms by which silencing ATG4B suppresses tumors. In addition, the dependency of tumor suppression on the proteolytic activity of ATG4B is not clear. In this study, our findings reveal the following. First, knockdown of ATG4B arrested the cell cycle at $G_{1}$ phase in several types of cancer cells, although not in LKB-1 null HeLa cells. Second, silencing ATG4B inhibited autophagy, reduced ATP levels and increased AMPK phosphorylation in the cells. Treatment with the AMPK inhibitor compound C diminished the $\mathrm{G}_{1}$ phase arrest caused by knockdown of ATG4B. Third, overexpression of the dominant negative mutant $\mathrm{ATG}^{\mathrm{B}} \mathrm{B}^{\mathrm{C} 74 \mathrm{~A}}$ increased AMPK phosphorylation and arrested cell cycle progression, suggesting that the genetic and pharmacological ablation of ATG4B might trigger the LKB1-AMPK energy-sensing pathway to block cell cycle progression at the $\mathrm{G}_{1}-\mathrm{S}$ phase transition in cancer cells.

ATG4B is the most active member of the ATG4 family and is responsible for MAP1LC3/ GABARAP conjugation and deconjugation, facilitating autophagy in mammalian cells. ATG4 knockdown or overexpression of an ATG4B catalytic mutant impairs both the deconjugation of MAP1LC3/GABARAP orthologs and autophagosome maturation, which may, in turn, block autophagosome fusion with lysosomes [6, 28, 29]. Knockdown of ATG4B increases the MAP1LC3-II levels and GFP-MAP1LC3 puncta to suppress autophagic flux in breast cancer cells $[10,12]$. In accordance with previous studies, our results show that knockdown of ATG4B increased the levels of MAP1LC3-II and GFP-MAP1LC3 puncta in cancer cells. Similar effects of increased MAP1LC3-II and decreased ATP levels were also observed in cells ectopically expressing ATG4B ${ }^{\mathrm{C} 74 \mathrm{~A}}$. These results imply that targeting ATG4B may initially attenuate autophagy and then trigger the energy-sensing pathway to induce cell cycle arrest. However, knockdown of ATG4B induced autophagic flux in colorectal cancer cells [10] likely due to alternative routes for autophagy modulation and the complicated nature of these routes in various types of cancer when ATG4B is targeted. In addition, overexpression of either $\mathrm{ATG}^{\mathrm{B}} \mathrm{B}^{\mathrm{WT}}$ or $\mathrm{ATG}^{\mathrm{A}} \mathrm{B}^{\mathrm{C} 74 \mathrm{~A}}$ reduces levels of the ATG7-MAP1LC3 intermediate in cells [29], indicating that excess ATG4B might result in MAP1LC3-I accumulation. Our current results also show that the overexpression of either the $\mathrm{ATG}^{4} \mathrm{~B}^{\mathrm{WT}}$ or $\mathrm{ATG} 4 \mathrm{~B}^{\mathrm{C} 74 \mathrm{~A}}$ mutant caused MAP1LC3-I accumulation in the cells, while silencing ATG4B reduced levels of the MAP1LC3-I form in the cells. These results raise the possibility that an autophagy-independent function of ATG4B might be involved in tumor proliferation. Therefore, verifying the dependence of autophagic flux on silencing ATG4B-induced cell cycle arrest may require further study.

AMPK is activated and serves as an energy sensor to control cell cycle progression and autophagy in cells under nutrient deprivation or stressed conditions [30,31]. AMPK not only directly phosphorylates cyclin-dependent kinase inhibitor p $27^{\text {kip } 1}$ at Thr198 to stabilize $\mathrm{p} 27^{\mathrm{kip} 1}$ in the nucleus but also indirectly activates Fox03a to induce $\mathrm{p} 27^{\mathrm{kip} 1}$ expression, which, in turn, arrests the cell cycle at $\mathrm{G}_{1}$ phase $[25,30]$. p2 $7^{\mathrm{kip} 1}$ expression was increased in ATG4Bsilenced cancer cells with and without proteasomal and lysosomal inhibitor treatment, implying that the transcriptional regulation of $\mathrm{p} 27^{\mathrm{kip} 1}$ may be increased in ATG4B knockdown cells. In addition, mTORC1 is a nutrient-responsive regulator of cell proliferation and is repressed by AMPK through either the phosphorylation of raptor [24] or the inhibition of Rheb [32], a GTP-binding protein required for mTORC1 activation. mTOR decreases CCND1 translation and results in cell cycle arrest at $G_{1}$ phase $[33,34]$. Combining these data with the results from our study, we propose that knockdown of ATG4B may transiently block autophagy to decrease ATP levels and provide feedback to activate AMPK for cell cycle arrest.

Several studies have revealed that ATG4B is highly expressed in cancer patients and that silencing ATG4B suppresses tumor growth in vitro and in a xenografted mouse model [10-12]. However, to date, the detailed mechanisms for the tumor-suppressive effects of ATG4B knockdown in cancer cells remain unclear. Our data show that silencing ATG4B 
might activate the LKB1-AMPK pathway to arrest the cell cycle at $\mathrm{G}_{1}$ phase in cancer cells. Although AMPK might be activated by several other kinases, such as $\mathrm{Ca}(2+)$ /calmodulindependent kinase (CAMKK2)[35] and mixed-lineage kinases 3 (MLK3) [36], our findings suggest that AMPK might be a suitable marker for tumor-suppressive effects in cancer cells when ATG4B is targeted. Moreover, AMPK is involved in sensitization of cancer cells to chemotherapeutic drugs $[37,38]$, implying that combinational treatment with ATG4B inhibitor and chemotherapeutic drugs might provide a potential cancer therapy in the future.

\section{Abbreviations}

ATG (autophagy-related); MAP1, MAP1LC3 (microtubule-associated proteins 1A/1B/1C light chain 3); AMPK (AMP-activated protein kinase); LKB1 (liver kinase B1); p27 ${ }^{\text {kip1 }}$ (cyclindependent kinase inhibitor 1B); CCND1 (cyclin D1); MTOR (mechanistic target of rapamycin); CQ (chloroquine); PE (phosphatidylethanolamine).

\section{Acknowledgements}

We thank Dr. Junying Yuan for providing the GFP-MAP1LC3 plasmid. This work was supported by the Ministry of Science and Technology (106 and 105-2311-B-075B-001), Kaohsiung Veterans General Hospital (VGHKS105-136, 104-G01-4), and the National Sun Yat-Sen University-KMU Joint Research Project (NSYSUKMU 106-P001).

\section{Disclosure Statement}

The authors declare no Disclosure Statement.

\section{References}

1 Nakatogawa H, Ichimura Y, Ohsumi Y: Atg8, a ubiquitin-like protein required for autophagosome formation, mediates membrane tethering and hemifusion. Cell 2007;130:165-178.

-2 Marino G, Uria JA, Puente XS, Quesada V, Bordallo J, Lopez-Otin C: Human autophagins, a family of cysteine proteinases potentially implicated in cell degradation by autophagy. J Biol Chem 2003;278:3671-3678.

-3 Li M, Hou Y, Wang J, Chen X, Shao ZM, Yin XM: Kinetics comparisons of mammalian Atg4 homologues indicate selective preferences toward diverse Atg8 substrates. J Biol Chem 2011;286:7327-7338.

-4 Shu CW, Drag M, Bekes M, Zhai D, Salvesen GS, Reed JC: Synthetic substrates for measuring activity of autophagy proteases: autophagins (Atg4). Autophagy 2010;6:936-947.

-5 Tanida I, Sou YS, Ezaki J, Minematsu-Ikeguchi N, Ueno T, Kominami E: HsAtg4B/HsApg4B/autophagin-1 cleaves the carboxyl termini of three human Atg8 homologues and delipidates microtubule-associated protein light chain 3- and GABAA receptor-associated protein-phospholipid conjugates. J Biol Chem 2004;279:36268-36276.

- 6 Yu ZQ, Ni T, Hong B, Wang HY, Jiang FJ, Zou S, Chen Y, Zheng XL, Klionsky DJ, Liang Y, Xie Z: Dual roles of Atg8-PE deconjugation by Atg4 in autophagy. Autophagy 2012;8:883-892.

- 7 Satoo K, Noda NN, Kumeta H, Fujioka Y, Mizushima N, Ohsumi Y, Inagaki F: The structure of Atg4BLC3 complex reveals the mechanism of LC3 processing and delipidation during autophagy. EMBO J 2009;28:1341-1350.

-8 Yang Z, Wilkie-Grantham RP, Yanagi T, Shu CW, Matsuzawa S, Reed JC: ATG4B (Autophagin-1) phosphorylation modulates autophagy. J Biol Chem 2015;290:26549-26561.

>9 Bortnik S, Choutka C, Horlings HM, Leung S, Baker JH, Lebovitz C, Dragowska WH, Go NE, Bally MB, Minchinton AI, Gelmon KA, Gorski SM: Identification of breast cancer cell subtypes sensitive to ATG4B inhibition. Oncotarget 2016;7. Doi:10.18632/oncotarget.11408 


\section{Cellular Physiology Cell Physiol Biochem 2017;44:728-740

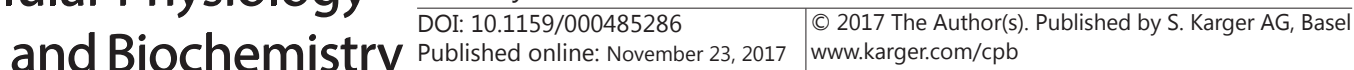

10 Liu PF, Leung CM, Chang YH, Cheng JS, Chen JJ, Weng CJ, Tsai KW, Hsu CJ, Liu YC, Hsu PC, Pan HW, Shu CW: ATG4B promotes colorectal cancer growth independent of autophagic flux. Autophagy 2014;10:14541465.

11 Rothe K, Lin H, Lin KB, Leung A, Wang HM, Malekesmaeili M, Brinkman RR, Forrest DL, Gorski SM, Jiang $\mathrm{X}$ : The core autophagy protein ATG4B is a potential biomarker and therapeutic target in CML stem/ progenitor cells. Blood 2014;123:3622-3634.

12 Bortnik S, Choutka C, Horlings HM, Leung S, Baker JH, Lebovitz C, Dragowska WH, Go NE, Bally MB, Minchinton AI, Gelmon KA, Gorski SM: Identification of breast cancer cell subtypes sensitive to ATG4B inhibition. Oncotarget 2016;7:66970-66988.

13 Toshima T, Shirabe K, Matsumoto Y, Yoshiya S, Ikegami T, Yoshizumi T, Soejima Y, Ikeda T, Maehara Y: Autophagy enhances hepatocellular carcinoma progression by activation of mitochondrial beta-oxidation. J Gastroenterol 2014;49:907-916.

14 Demeulder B, Zarrinpashneh E, Ginion A, Viollet B, Hue L, Rider MH, Vanoverschelde JL, Beauloye C, Horman S, Bertrand L: Differential regulation of eEF2 and p70S6K by AMPKalpha2 in heart. Biochim Biophys Acta 2013;1832:780-790.

15 DeSelm CJ, Miller BC, Zou W, Beatty WL, van Meel E, Takahata Y, Klumperman J, Tooze SA, Teitelbaum SL, Virgin HW: Autophagy proteins regulate the secretory component of osteoclastic bone resorption. Dev Cell 2011;21:966-974.

16 Marino G, Fernandez AF, Cabrera S, Lundberg YW, Cabanillas R, Rodriguez F, Salvador-Montoliu N, Vega JA, Germana A, Fueyo A, Freije JM, Lopez-Otin C: Autophagy is essential for mouse sense of balance. J Clin Invest 2010;120:2331-2344.

17 Read R, Savelieva K, Baker K, Hansen G, Vogel P: Histopathological and neurological features of Atg4b knockout mice. Vet Pathol 2011;48:486-494.

-18 Kanamori H, Takemura G, Maruyama R, Goto K, Tsujimoto A, Ogino A, Li L, Kawamura I, Takeyama T, Kawaguchi T, Nagashima K, Fujiwara T, Fujiwara H, Seishima M, Minatoguchi S: Functional significance and morphological characterization of starvation-induced autophagy in the adult heart. Am J Pathol 2009;174:1705-1714.

19 Tang AH, Rando TA: Induction of autophagy supports the bioenergetic demands of quiescent muscle stem cell activation. EMBO J 2014;33:2782-2797.

20 Hardie DG, Ross FA, Hawley SA: AMPK: a nutrient and energy sensor that maintains energy homeostasis. Nat Rev Mol Cell Biol 2012;13:251-262.

-21 Shaw RJ, Kosmatka M, Bardeesy N, Hurley RL, Witters LA, DePinho RA, Cantley LC: The tumor suppressor LKB1 kinase directly activates AMP-activated kinase and regulates apoptosis in response to energy stress. Proc Natl Acad Sci U S A 2004;101:3329-3335.

22 Soliman GA: The role of mechanistic target of rapamycin (mTOR) complexes signaling in the immune responses. Nutrients 2013;5:2231-2257.

23 Hay N, Sonenberg N: Upstream and downstream of mTOR. Genes Dev 2004;18:1926-1945.

-24 Gwinn DM, Shackelford DB, Egan DF, Mihaylova MM, Mery A, Vasquez DS, Turk BE, Shaw RJ: AMPK phosphorylation of raptor mediates a metabolic checkpoint. Mol Cell 2008;30:214-226.

-25 Liang J, Shao SH, Xu ZX, Hennessy B, Ding Z, Larrea M, Kondo S, Dumont DJ, Gutterman JU, Walker CL, Slingerland JM, Mills GB: The energy sensing LKB1-AMPK pathway regulates p27(kip1) phosphorylation mediating the decision to enter autophagy or apoptosis. Nat Cell Biol 2007;9:218-224.

-26 Hyun SY, Jang YJ: p53 activates G(1) checkpoint following DNA damage by doxorubicin during transient mitotic arrest. Oncotarget 2015;6:4804-4815.

27 Walerych D, Napoli M, Collavin L, Del Sal G: The rebel angel: mutant p53 as the driving oncogene in breast cancer. Carcinogenesis 2012;33:2007-2017.

-28 Betin VM, Singleton BK, Parsons SF, Anstee DJ, Lane JD: Autophagy facilitates organelle clearance during differentiation of human erythroblasts: Evidence for a role for ATG4 paralogs during autophagosome maturation. Autophagy 2013;9:

-29 Fujita N, Hayashi-Nishino M, Fukumoto H, Omori H, Yamamoto A, Noda T, Yoshimori T: An Atg4B mutant hampers the lipidation of LC3 paralogues and causes defects in autophagosome closure. Mol Biol Cell 2008;19:4651-4659. 


\section{Cellular Physiology Cell Physiol Biochem 2017;44:728-740 \begin{tabular}{l|l|l} 
DOI: 10.1159/000485286 & $\begin{array}{l}\text { O 2017 The Author(s). Published by S. Karger AG, Basel } \\
\text { www.karger.com/cpb }\end{array}$ \\
\hline
\end{tabular} \\ Liu et al.: AMPK Activation in ATG4B-Knockdown Cancer Cells}

-30 Egan DF, Shackelford DB, Mihaylova MM, Gelino S, Kohnz RA, Mair W, Vasquez DS, Joshi A, Gwinn DM, Taylor R, Asara JM, Fitzpatrick J, Dillin A, Viollet B, Kundu M, Hansen M, Shaw RJ: Phosphorylation of ULK1 (hATG1) by AMP-activated protein kinase connects energy sensing to mitophagy. Science 2011;331:456461.

31 Du L, Shen T, Liu B, Zhang Y, Zhao C, Jia N, Wang Q, He Q: Shock Wave Therapy Promotes Cardiomyocyte Autophagy and Survival during Hypoxia. Cell Physiol Biochem 2017;42:673-684.

-32 Lacher MD, Pincheira RJ, Castro AF: Consequences of interrupted Rheb-to-AMPK feedback signaling in tuberous sclerosis complex and cancer. Small GTPases 2011;2:211-216.

-33 Shaw RJ: LKB1 and AMP-activated protein kinase control of mTOR signalling and growth. Acta Physiol (Oxf) 2009;196:65-80.

34 Averous J, Fonseca BD, Proud CG: Regulation of cyclin D1 expression by mTORC1 signaling requires eukaryotic initiation factor 4E-binding protein 1 Oncogene 2008;27:1106-1113.

35 Fogarty S, Ross FA, Vara Ciruelos D, Gray A, Gowans GJ, Hardie DG: AMPK Causes Cell Cycle Arrest in LKB1Deficient Cells via Activation of CAMKK2. Mol Cancer Res 2016;14:683-695.

-36 Luo L, Jiang S, Huang D, Lu N, Luo Z: MLK3 phophorylates AMPK independently of LKB1. PLoS One 2015;10 Doi:10:e0123927.

37 Chen TJ, Zhou YF, Ning JJ, Yang T, Ren H, Li Y, Zhang S, Chen MW: NBM-T-BMX-OS01, an Osthole Derivative, Sensitizes Human Lung Cancer A549 Cells to Cisplatin through AMPK-Dependent Inhibition of ERK and Akt Pathway. Cell Physiol Biochem 2015;36:893-906.

-38 Yun M, Lee D, Park MN, Kim EO, Sohn EJ, Kwon BM, Kim SH: Cinnamaldehyde derivative (CB-PIC) sensitizes chemo-resistant cancer cells to drug-induced apoptosis via suppression of MDR1 and its upstream STAT3 and AKT signalling. Cell Physiol Biochem 2015;35:1821-1830. 